\title{
RELACIÓN ENTRE LA CONDICIÓN CORPORAL Y EL NIVEL DE INFESTACIÓN PARASITARIA EN BOVINOS A PASTOREO COMO CRITERIO PARA EL TRATAMIENTO ANTIHELMÍNTICO SELECTIVO
}

\author{
Relationship Between Body Condition and Level of Parasite Infestation in \\ Grazing Cattle as a Criterion for Selective Deworming
}

\author{
Gustavo Morales ${ }^{1,2}$, Luz Arelis Pino', Espartaco Sandoval ${ }^{3}$, Delia Jiménez ${ }^{4}$, \\ Jairo Morales $^{5}$
}

\section{RESUMEN}

\begin{abstract}
El objetivo del trabajo consistió en evaluar la relación entre el nivel de infestación por parásitos tipo estróngilos y la condición corporal en bovinos Criollo Río Limón $(\mathrm{n}=42)$, Bos taurus $(\mathrm{n}=54)$ y Bos indicus $(\mathrm{n}=11)$, pertenecientes a dos fincas ganaderas del Municipio Pedraza, Estado Barinas (Venezuela). Las muestras de heces se analizaron por la técnica cuantitativa de McMaster con solución salina sobresaturada. Se evaluó la condición corporal (CC) con una escala de 1 a 5 ( 1 = flaco; 5 = obeso) empleando el grado de 2.5 como punto de inflexión. Los animales fueron clasificados como resilientes (CC>2.5 $\mathrm{y}$ alto recuento de huevos por gramo de heces [ $>800]$ ), resistentes (CC $>2.5$ y carga parasitaria nula, leve o moderada), sensibles (CC $<2.5$, alta carga parasitaria) y falso problema parasitario (CC $<2.5$ y carga parasitaria baja o nula). Los bovinos Criollo Río Limón se asociaron con las categorías de resiliente y resistente, los Bos taurus con las categorías de sensibles y falso problema parasitario, y los Bos indicus con la categoría de resistente. La evidente relación entre condición corporal y nivel de la infestación por parásitos tipo estróngilos permite realizar una eficiente y rápida selección de la fracción de bovinos a ser tratados con antihelmínticos.
\end{abstract}

Palabras clave: raza, condición corporal, resistente, resiliente, sensible, selección, tratamiento selectivo

\section{Abstract}

The aim of this study was to evaluate the relationship between the level of Strongyletype parasite infestation and body condition in Criollo Rio Limon $(n=42)$, Bos taurus

\footnotetext{
${ }^{1}$ Instituto Nacional de Investigaciones Agrícolas, CENIAP, Maracay, Venezuela

${ }^{2}$ E-mail: gustavomoralesc@gmail.com,gmorales@canaima2.inia.gob.ve

${ }^{3}$ INIA, Centro de Investigaciones Agrícolas del Estado Yaracuy, Venezuela E-mail: esandoval@canaima2.inia.gob.ve

${ }^{4}$ Docente agropecuario en ejercicio libre

${ }^{5}$ Núcleo Universitario “Rafael Rangel”, Departamento de Ciencias Agrarias, Universidad de Los Andes, Trujillo, Venezuela
} 
$(\mathrm{n}=54)$ and Bos indicus $(\mathrm{n}=11)$. The cattle belonged to two farms at Pedraza municipality in Barinas State (Venezuela). Fecal samples were analyzed by the quantitative McMaster technique using saturated saline solution. Body condition score (BCS) was evaluated using a scale of 1 to 5 ( $1=$ thin, $5=$ obese $)$ where 2.5 was the cut-off point. The animals were classified as resilient (BCS $>2.5$ and high egg counts per gram of feces $[>800]$ ), resistant (BCS $>2.5$ and null, mild or moderate parasite burden), sensitive (BCS $<2.5$, high parasite burden), and false parasite problem (BCS $<2.5$ and low or no parasite burden). The Criollo Río Limón was associated with resilient and resistant categories, the Bos taurus to the sensitive and false parasite problem categories, and Bos indicus to the resistant category. The relationship between body condition and level of Strongyle-type parasite infestation allows an efficient and rapid identification of the fraction of cattle requiring treatment with anthelmintics.

Key words: breed, body condition, resistant, resilient, sensible, selection, selective treatment

\section{INTRODUCCIÓN}

La estrongilosis gastrointestinal o gastroenteritis parasitaria de los rumiantes es debida a la presencia en el abomaso, intestino delgado e intestino grueso de nematodos pertenecientes al orden Strongylida y, que pese a estar ubicados en varias familias, se agrupan bajo la denominación de tipo estróngilos. La estrongilosis gastrointestinal es considerada una parasitosis cosmopolita (Morales y Pino, 2009).

Los géneros más comunes en Venezuela pertenecen a las familias Trichostrongylidae (Haemonchus, Mecistocirrus, Trichostrongylus, Cooperia, Ostertagia, Teladorsagia); Ancylostomatidae (Bunostomum, Agriostomum) y Strongylidae (Oesophagostomum). Los ciclos biológicos de estos parásitos son bastante similares, ya que son directos; es decir, no requieren de hospederos intermediarios para completar sus ciclos de vida.

Los niveles de infestación parasitaria se asocian con manifestaciones clínicas, tales como anorexia, anemia, diarrea y caquexia (Graber y Perrotin, 1983; Urquhart et al., 1999). Los signos clínicos pueden pasar desapercibidos en casos de infestaciones leves o medias o ser confundidos con problemas nutricionales o de manejo; sin embargo, po- drían servir como criterio de apoyo para el tratamiento antihelmíntico selectivo en fincas con antecedentes de problemas parasitarios. Se dispone de evidencias que la distribución de las cargas parasitarias al interior del rebaño no es uniforme y que aunque pastoreen los mismos potreros y pertenezcan a grupos de edad similares, sólo un pequeño porcentaje de hospedadores alberga el mayor número de especies y cargas parasitarias (Morales, 1989; Morales et al., 1998; Morales y Pino, 2003); de allí que el tratamiento selectivo de la fracción de animales que lo amerite debería constituir una acertada estrategia de control (Morales et al., 2006a,b; 2007, 2008).

Para Eddi (1996), el empleo mínimo y eficiente de los antiparasitarios al realizar desparasitaciones estratégico/racionales constituyen una buena alternativa de control. El control parasitario basado en estrategias que se oponen al tratamiento en masa de los rebaños está sustentado en estudios que han demostrado los efectos nocivos del uso inadecuado de los antihelmínticos, tales como la presencia de residuos tóxicos en los tejidos como consecuencia del uso de principios activos de larga persistencia (Vercruysse y Dorny, 1999), el efecto sobre el medio ambiente por actuar negativamente sobre la fauna edáfica, lo cual se encuentra bien documentado para el caso de las ivermectinas (Fiel et al., 2001). Asimismo, la utilización indiscriminada y prolongada, así como la 
elevada frecuencia de tratamientos empleando el mismo principio activo constituye la principal causa de aparición de cepas de parásitos quimioresistentes (Fiel et al., 2001), ocasionando la reducción del arsenal terapéutico disponible para la lucha contra los helmintos gastrointestinales que afectan a los rumiantes (Strong, 1993; Morales et al., 1998; FAO, 2003; Romero, 2005).

El recuento de huevos de helmintos en heces y las necropsias son de gran valor cuando se dispone de laboratorio y personal especializado, pero muchos productores carecen del acceso a estas facilidades, de allí que el objetivo del presente ensayo fue evaluar el uso de la condición corporal como criterio de base en la implementación de tratamientos antihelmínticos selectivos.

\section{Materiales y Métodos}

\section{Fincas y Criterios de Inclusión}

El trabajo se realizó en el campo experimental “Ciudad Bolivia”, del Instituto Nacional de Investigaciones Agrícolas (INIA) $\mathrm{y}$ en una finca comercial, ambas ubicadas en el Estado Barinas, Venezuela. La precipitación promedio anual fue de $1840 \mathrm{~mm}$, temperatura de $26.5^{\circ} \mathrm{C}$, evaporación máxima de $249 \mathrm{~mm}$ y la humedad relativa alcanza 84\% en el periodo de lluvias y 78\% en la época seca (Lugo et al., 2006).

El campo experimental disponía de potreros con yaragua (Hyparrenia rufa), pasto alemán (Echinochloa polystachia), brizanta (Brachiaria brizantha), decumbens (Brachiaria decumbens) y guinea (Panicum máximum) y la finca comercial tenía pasto brizanta, decumbens y pasto alemán. El tipo de crianza en ambas fincas fue semi-intensivo, donde los bovinos adultos reciben suplementación con sales minerales y los becerros alimento concentrado. Asimismo, el control del parasitismo gastrointestinal se lleva a cabo con el uso de antihelmínticos comerciales, donde la dosis se establece por estimación visual del peso de los animales.
Se trabajó con animales de 12 a 24 meses de edad, con no menos de 6 meses de pastoreo, a fin de garantizar que hayan estado expuestos a larvas infectantes (L3) en los potreros. Los animales debían haber recibido al menos un tratamiento antiparasitario y haber tenido la oportunidad de reinfectarse en condiciones naturales (solo se incluyeron animales con tres o más meses de desparasitados). En el campo experimental se trabajó con 42 animales Criollo Río Limón $(\mathrm{n}=42)$ y en la finca comercial con 11 de tipo Brahman (Bos indicus), 27 cruces de $1 / 2$ Bos taurus $\mathrm{x}$ $1 / 2$ tipo Brahman y 27 animales con predominancia de Bos taurus (Holstein, Pardo Suizo y Carora). Los animales cruzados fueron considerados como un solo tipo racial, y se les denominó Bos taurus en el análisis estadístico.

En el campo experimental, el tratamiento antihelmíntico (ivermectina o albendazole) se hacía dos veces al año para los adultos y cada cuatro meses para los jóvenes. Además, las vacas eran dosificadas con albendazole 30 días antes del parto. Estos antihelmínticos se empleaban en la finca desde hace tres años. En la finca comercial, el tratamiento en los adultos se hacía con con ivermectina dos veces al año y en los jóvenes con levamisol (oral) en forma trimestral, y estos productos se empleaban en la finca desde hace cinco años.

\section{Evaluación Parasitológica}

Se colectó muestras de heces tomadas directamente del recto y las muestras se analizaron con la técnica cuantitativa de McMaster y una solución salina sobresaturada como líquido de flotación. Los niveles de infestación por estróngilos se establecieron de acuerdo a Hansen y Perry (1994) y Morales y Pino (2009) y se expresaron en huevos por gramo de heces (hpg). Se consideró como una carga leve a animales con 50 a 200 hpg, con carga moderada con $>200$ y $<800$ hpg, y con carga alta con $>800 \mathrm{hpg}$.

Los animales, según la carga parasitaria fueron clasificados, según FAO (2003) 
Cuadro 1. Criterios para la clasificación de los bovinos considerando la condición corporal y el nivel de infestación por estróngilos, expresados en recuentos de huevos por gramo de heces (hpg)

\begin{tabular}{lcl}
\hline Clasificación & Condición Corporal & Nivel de Infección ${ }^{1}$ \\
\hline Resistente & $>2.5$ & Negativo, leve o moderado \\
"Resiliente" & $>2.5$ & Alto \\
Acumulador de parásitos o sensible & $<2.5$ & Alto \\
Falso problema parasitario & $<2.5$ & Leve o negativo \\
\hline
\end{tabular}

${ }^{1}$ Leve: 50 a 200; Moderado: $>200$ a $\leq 800$ : Alto: $>800$ hpg

como "Resistentes" (animales con baja carga parasitaria) y "Resilientes" (habilidad de mantener niveles productivos aceptables $\mathrm{y}$ apariencia saludable a pesar de presentar altas cargas parasitarias). Asimismo, se incluye la categoría de "Acumulador de Parásitos" o "Sensible" (hospedadores con altas cargas parasitarias y manifestación de signos clínicos de parasitismo), según Morales et al. (1998), y se introduce una nueva categoría denominada "Falso problema parasitario" (referido a animales con pobre condición corporal $(<2.5)$ con baja carga o negativos a la infección por parásitos gastrointestinales) (ver Cuadro 1).

Además, se realizó una estimación de la contaminación de la pastura a través de una coproscopía cuantitativa con la técnica de McMaster (Hansen y Perry, 1994; Morales y Pino, 2009).

\section{Condición Corporal}

La condición corporal se evaluó de acuerdo el método sugerido por Martínez et al. (1998), modificado y ajustado por el número de costillas visibles y por la observación y palpación de la zona lumbar, nacimiento de la cola, cadera y grupa. Se empleó una escala de de 0 a 5 donde 1 es muy delgado y
5 es obeso, con 2.5 como punto de inflexión. En términos generales, animales con escalas menores de 2.5 fueron aquellos donde se observaban las apófisis transversas de las vértebras lumbares y las últimas costillas, además del vaciado de la grupa.

\section{Análisis Estadístico}

En vista que la raza de los animales era diferente en las fincas en estudio, se comparó la contaminación de los potreros con huevos tipo estróngilos mediante la prueba no paramétrica de Mann y Whitney (Morales y Pino, 2009), para determinar la posibilidad de un efecto confundido por efecto del grupo racial con la finca.

Se hizo un análisis discriminante lineal con la finalidad de validar la clasificación realizada a priori y reubicar a aquellos animales que resultaron mal ubicados. Luego, la información obtenida fue organizada en una tabla de contingencia entre la clasificación de resistente, "resiliente", sensible y falso problema parasitario (FPP) y el tipo racial (Bos taurus, Bos indicus y Criollo Río Limón). Con la información contenida en las tablas de datos categorizadas se realizó un análisis de correspondencia simple. Todos los análisis fueron realizados con InfoStat (2004).

\footnotetext{
${ }^{6}$ Término de reciente introducción en parasitología veterinaria por Castell (2002), adaptado de acuerdo al Diccionario Enciclopédico Salvat (Salvat Editores, 1973) y citado por FAO (2003).
} 


\section{Resultados}

La contaminación ambiental con huevos de estróngilos es dependiente de factores vinculados al hospedador, factores tales como el estado inmunológico, la carga parasitaria y la carga animal del potrero (Urquhart et al., 1999). Los recuentos de hpg pueden dar un estimado del nivel de contaminación y del posible grado de infectividad de las pasturas (Fiel et al., 1998), de ahí que la coproscopía cuantitativa constituye la medida indirecta más corrientemente utilizada para dicha evaluación (Mandonnet, 1995). No obstante, se debe enfatizar que esta evaluación es diferente de la estimación del potencial infectante de la pastura, ya que esta última requiere de la cuantificación de las larvas infectantes (L3) por kilogramo de pasto.

La contaminación de los potreros con huevos de estróngilos fue similar en ambas explotaciones ganaderas (Cuadro 2). La Fig. 1 muestra que los Bos taurus se asociaron principalmente con las condiciones de sensible y FPP, los Bos indicus con la condición de resistentes, y el Criollo Río Limón con las condiciones de resistente y de resiliencia. $\mathrm{La}$ condición de resiliencia obtuvo la menor frecuencia.

El Cuadro 3 muestra el nivel de parasitismo causado por estróngilos (medido en número de huevos por gramo de heces) en base a los grupos considerados como resiliente, resistente, sensible y de falso problema parasitario, donde el 81.8\% (9/11) de Bos indicus, 38.8\% (21/54) de Bos taurus y 59.5\% (25/42) de los Criollo Río Limón clasificaron como resistentes, mientras que los sensibles y falso problema parasitario correspondieron mayoritariamente a Bos taurus.

\section{Discusión}

La posibilidad de un efecto confundido entre el grupo racial y la finca quedó descartada al demostrarse que las pasturas de am bas fincas se contaminaban con similares niveles de parásitos tipo estróngilos.

El ganado tipo Brahman en las condiciones de Venezuela es, en realidad, el producto de una mezcla de razas cebú, y donde los animales estuvieron expuestos por generaciones a deficiente alimentación, altas cargas parasitarias y soportando rigores climáticos extremos, que los habilitaron para sobrevivir y producir con limitada eficiencia (Valle, 2008b).

La mayor resistencia a la infestación por nematodos gastrointestinales mostrada por el Bos indicus en relación al Bos taurus se encuentra bien documentada (Le Jambre, 1982; Peña et al., 2000; Morales et al., 2001). Como ejemplo se puede citar el caso de la raza Nelore, que muestra mayor resistencia a la infestación por nematodos gastrointestinales (Bricarello et al., 2007), a la infección por Trypanosoma sp. (Sandoval et al., 1998) y a los ectoparásitos; razón por la cual los criadores locales realizan cruzamientos empíricos con animales Bos indicus para disminuir la dependencia a los acaricidas y la incidencia de enfermedades trasmitidas por garrapatas (FAO, 2003).

La mayor susceptibilidad del Bos taurus a las infestaciones parasitarias es ampliamente conocida (Urquhart et al., 1999). En el presente estudio, la pobre condición corporal, asociada en parte a sus problemas de adaptación al medio (O’Kelly, 1980), y su mayor susceptibilidad al parasitismo gastrointestinal determinaron que la mayor parte de los animales estuvieran asociados a la categoría de sensibles.

Si bien es cierta la mayor adaptabilidad y resistencia de las razas cebuínas frente a las razas europeas, dichas diferencias también existen dentro de individuos al interior de las razas (Morales et al., 2001), demostrando que la intensidad de la misma es significativamente dependiente del grado de respuesta inmune (Meeusen, 1999). En el presente estudio se pudo identificar animales 
Cuadro 2. Comparación de la contaminación de los potreros con huevos tipo estróngilos (huevos por grano de heces $-\mathrm{hpg}$ ) en dos explotaciones ganaderas del Estado Barinas, Venezuela

\begin{tabular}{lccc}
\hline Finca & $\begin{array}{c}\text { Bovinos } \\
(\mathrm{n})\end{array}$ & Media & Mediana \\
\hline Finca comercial & 65 & 467.7 & 200 \\
Campo exp erimental & 42 & 433.3 & 100 \\
\hline
\end{tabular}

$p=0.19$ (no significativo)

Cuadro 3. Carga parasitaria promedio (huevos por gramo de heces $-\mathrm{hpg}$ ) en bovinos clasificados en base a su condidión infectación parasitaria (resiliente, resistente, sensible y de falso problema parasitario)

\begin{tabular}{lcccccccc}
\hline \multirow{2}{*}{ Grupo racial } & \multicolumn{2}{c}{$\begin{array}{c}\text { Falso problema } \\
\text { parasitario }\end{array}$} & Resiliente & \multicolumn{2}{c}{ Resistente } & \multicolumn{2}{c}{ Sensible } \\
\cline { 2 - 10 } & $\mathrm{n}$ & $\mathrm{hpg}$ & $\mathrm{n}$ & $\mathrm{hpg}$ & $\mathrm{n}$ & $\mathrm{hpg}$ & $\mathrm{n}$ & $\mathrm{hpg}$ \\
\hline Bos indicus & 1 & 50 & 0 & 0 & 9 & 128 & 1 & 1000 \\
Bos taurus & 17 & 91 & 1 & 1100 & 21 & 305 & 15 & 1277 \\
Cri ollo Río Limón & 7 & 12 & 5 & 2020 & 25 & 84 & 5 & 1180 \\
\hline
\end{tabular}

1 Ver Cuadro 1

FPP, sensibles y resistentes en los tres grupos raciales (Cuadro 3), lo cual destaca el carácter individual de la resistencia frente a la infestación parasitaria e implica la necesidad de evaluar este aspecto como criterio para el descarte o selección de reproductores y para la realización de tratamientos selectivos al interior del rebaño (Morales y Pino, 2009).

El Criollo Río Limón, en las condiciones del sur del lago Maracaibo en Venezuela, ha sido reportado con una menor prevalencia de parasitismo gastrointestinal que el ganado Pardo Suizo criado bajo las mismas condicio- nes (Moreno,1996). Esta raza presenta las características del bovino tropical, ya que está muy adaptado a las condiciones de pastoreo y los animales presentan alta rusticidad (Valle, 2008b). En este estudio se pudo constatar su adaptación al medio y la tolerancia al parasitismo gastrointestinal, ya que los resultados de condición corporal y los recuentos de huevos tipo estróngilos hicieron que la mayor parte de los animales hayan quedado dentro de las categorías de resistente y “resilientes”. Esta condición permite que requieran menos dosificaciones por periodo de tiempo que animales de otras razas en las mismas pasturas (Bisset y Morris, 1996; Sandoval et al., 2006). 


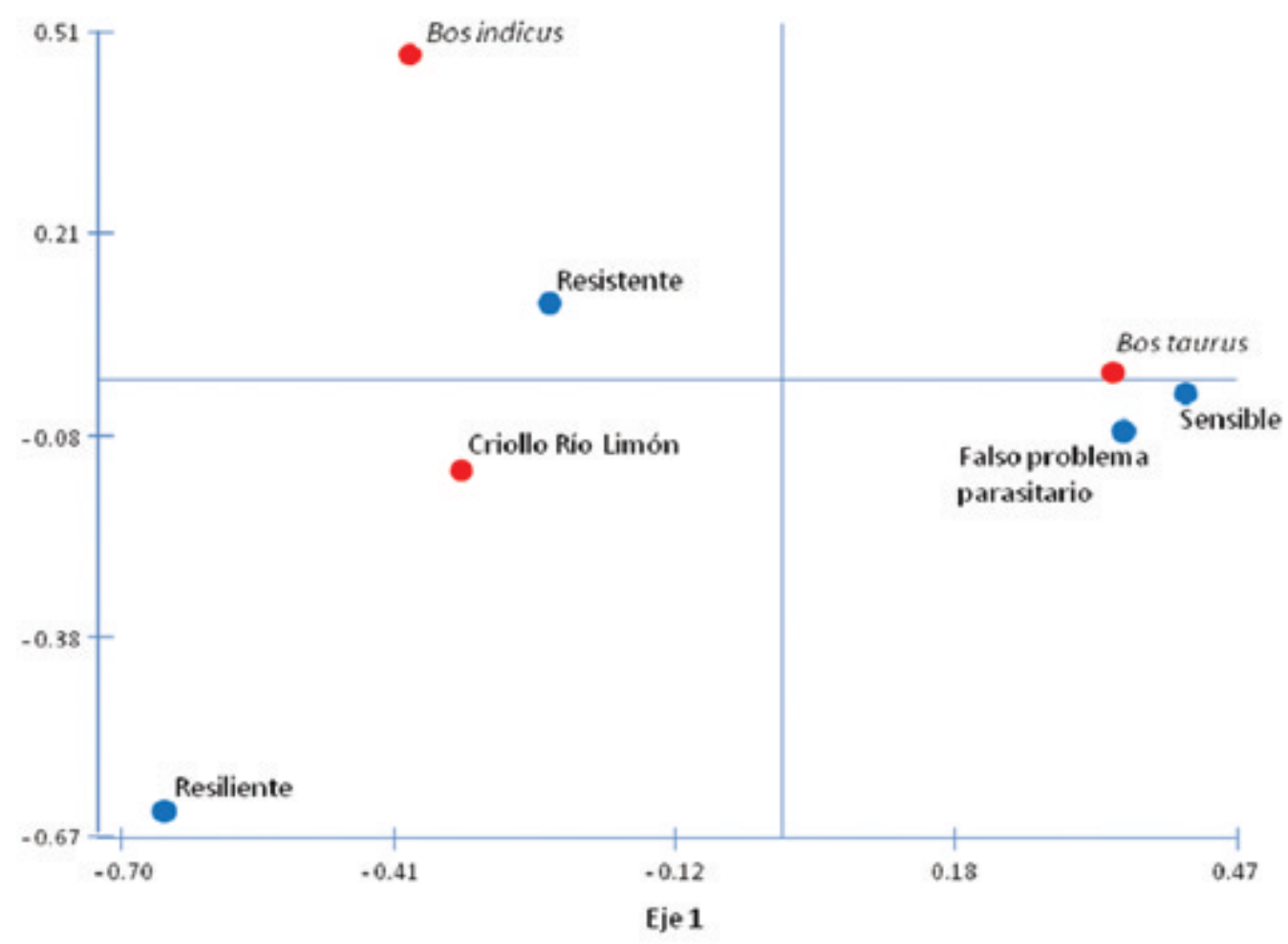

Figura 1. Análisis de correspondencia entre animales Bos taurus, Bos indicus y Criollo Río Limón y la condición de hospedador resistente, resilente, sensible y de falso problema parasitario

La resistencia a determinadas enfermedades, especialmente al parasitismo gastrointestinal es un carácter heredable. Hay evidencias que demuestran diferencias entre y dentro de razas. Por ejemplo, Angulo et al. (2010), en un estudio de comparación de estrategias de control antihelmíntico en terneras doble propósito encontraron que hubo animales que mantuvieron una buena condición corporal sin recibir tratamiento. Esta característica debería integrarse a los programas de selección genética o de cruzamientos, ya que los animales resistentes requieren un menor número de tratamientos al año y limitan la contaminación de los pastos (FAO, 2003).

El hecho de que animales criados bajo idénticas condiciones presenten diferente condición corporal permite inferir que aquellos con una buena condición corporal reflejan una mejor adaptabilidad al medio, y den- tro de esto se podría incluir la resistencia o tolerancia al parasitismo gastrointestinal.

La apreciación visual de la condición corporal tiene las ventajas de ser de fácil aprendizaje, económica, no requiere de personal especializado y puede aplicarse sin entrar en contacto con el animal (Ramírez, 2005). El uso de la condición corporal como criterio de selección de la fracción de animales a ser tratados con antihelmínticos es, al igual que en el método FAMACHA (Kaplan et al., 2004), una medida de resiliencia más que de resistencia. La condición corporal considera el deterioro del estado físico del animal como una manifestación del efecto infestación parasitaria, tal y como la detección de anemia por el método FAMACHA es considerada una manifestación del efecto Haemonchus en el ovino (Bisset, 2000; Malan et al., 2000; Morales et al., 2010). 
La condición corporal permite agrupar los animales de un rebaño dentro de dos grandes grupos: El primero que requiere tratamiento ( $<2.5)$, el cual concierne a los sensibles y a los falsos problemas parasitarios, y el segundo, formado por aquellos que no requieren tratamiento (>2.5) y que incluye a los resistentes y a los “resilientes". En ambos grupos se podrían encontrar animales con alto poder contaminante de la pastura, pero el tratamiento de los bovinos con condición corporal $<2.5$ ocasiona una importante reducción de dicha contaminación, además de liberar al hospedador de su carga parasitaria. Por otro lado, el no tratamiento de los animales "resilientes" permite el mantenimiento de poblaciones parasitarias en refugio (larvas en el pastizal aportadas por los animales no tratados) que se encargarían de "diluir" las poblaciones de nematodos resistentes, y de esa manera se disminuye el riesgo de aparición de cepas de parásitos quimioresistentes (VanWyk, 2001; FAO, 2003; Angulo et al., 2010).

Para Valle (2008a), al relacionar el índice de la condición corporal con la productividad, la alimentación, la salud, el bienestar y el manejo de los animales, se dota a los profesionales, técnicos y ganaderos de una herramienta sencilla pero de gran utilidad para una gestión eficiente de la unidad productiva. Esto es acorde con resultados del presente estudio, ya que permite realizar una selección rápida y eficiente de animales que al interior del rebaño requieren de tratamiento antihelmíntico, o para ser sometidos a un diagnóstico diferencial con respecto al parasitismo gastrointestinal y determinar si su baja condición corporal es una expresión de una alta infestación parasitaria, sin tener que realizar exámenes de laboratorio a todo el rebaño (Morales y Pino, 2009).

\section{Agradecimientos}

Los autores agradecen a la Ing. Jazmín Florio por la colaboración prestada durante el muestreo coprológico y en la evaluación de la condición corporal de los bovinos Criollo Río Limón de la Estación Experimental "Ciudad Bolivia”.

\section{Literatura Citada}

1. Angulo F, Parra A, Urdaneta A, Urdaneta M, Chacin E, Ramírez R. 2010. Efecto de diferentes estrategias de control antihelmíntico sobre nemaodos gastrointestinales en terneras doble propósito. Rev Científica, FCV-LUZ 20(6): 595-599.

2. Bisset S. 2000. Practical ways of implementing identification of host resistance in sheep and its use in breeding programmes. In: FAO TCP Workshop "Sustainable worm control programmes for sheep and goats". South Africa. p 16-21.

3. Bisset S, Morris C. 1996. Feasibility and implications of breeding sheep for resilience to nematode challenge. Int $\mathrm{J}$ Parasitol 26: 869:877.

4. Bricarello P, Zaros L, Coutinho L, Rocha $R$, Kooymann F, De Vries E, Goncalves J, Lima L, et al. 2007. Field study on nematodo resistance Nelorebreed cattle. Vet Parasitol 141: 291-301.

5. Castells D. 2002. Métodos alternativos para el control de endoparásitos: Uso de huéspedes resistentes. En: Reunión de especialistas en parasitología veterinaria de Argentina, Brasil, Chile y Uruguay.Facultad de Ciencias Veterinarias, Tandil, Argentina. [Internet]. Disponible en: http://www.inta.gov.ar/producto/ helminto/rtandil_06htm

6. Eddi C. 1996. Inmunidad, e inmuno profilaxis de las helmintiasis gastrointestinales de los bovinos. Tópicos de Parasitología Veterinaria 1(3): 43-51.

7. [FAO] Organización de las Naciones Unidades para la Alimentación y la Agricultura. 2003. Resistencia a los antiparasitarios. Estado actual con énfasis en América Latina. Estudio FAOProducción y Sanidad Animal № 157. Roma: FAO. 51 p.

8. Fiel C, Steffan P, Ferreyra D. 1998. Manual para el diagnóstico de nematodos en bovinos: Técnicas de frecuente utilización en la práctica veterinaria: su in- 
terpretación. Argentina: División de Sanidad Animal, Bayer Argentina. 61 p.

9. Fiel C, Ansían $O$, Suárez V, Vázquez $R$, Eddi C, Romero J, Caracostantogolo J, Saumell C, et al. 2001. Resistencia antihelmíntica en bovinos: causas, diagnóstico y profilaxis. Vet Argentina 18(171): 21-33.

10. Graber M, Perrotin C. 1983. Helminthes et helminthoses des ruminants domestiques d'Afrique tropicale. Maisons Alfort, Francia: Institut D’Elevage et de Médicine Vétérinaire des Pays Tropicaux. 378 p.

11. Hansen J, Perry B. 1994. The epidemiology, diagnosis and control of helminthes parasites of ruminants. Nairobi; International Laboratory for Research on Animal Diseases. $171 \mathrm{p}$.

12. InfoStat. 2004. InfoStat $v$ 2004. Argentina: Grupo InfoStat, FCA, Universidad Nacional de Córdoba.

13. Kaplan RM, Burke JM, Terrill TH, Miller JE, Getz WR, Mobini S, Valencia E, et al. 2004. Validation of the FAMACHA eye color chart for detecting clinical anemia in sheep and goats on farms in the southern United States. Vet Parasitol 123: 105-120.

14. LeJambre LF. 1982. Genetic and the control of trichostrongylid parasites of ruminants. In: Biology and control of endoparasites. Australia: Academic Press. p 53-80.

15. Lugo M, Florio J, Fuenmayor A, Pérez N, Sánchez E. 2006. Caracterización forrajera en fincas doble propósito del municipio Pedraza, estado Barinas. En: XIII Memorias Congreso Venezolano de Producción e Industria Animal. San Juan de los Morros, Venezuela: Universidad Rómulo Gallegos. p 195.

16. Malan FS, Van Wyk JA, Wessel CD. 2000. Clinical evaluation of anaemia in sheep: early trials. In: FAO TCP TCP Workshop "Sustainable worm control programmes for sheep and goats". South Africa. p 34-39.
17. Mandonnet N. 1995. Analyse de la variabilité génétique de la résistance aux strongles gastro-intestinaux chez les petits ruminants. Eléments pour la définition d'objectifs et de critères de sélection en milieu tempéré ou tropical. Thèse Docteur en Sciences. Orsay, France: Université de Paris. 115 p.

18. Martínez $N$, Herrera $P$, Birbe B, Domínguez C. 1998. Relación entre la condición corporal y la repuesta reproductiva de hembras bovinas de doble propósito. En: Madrid N, Soto E (eds). Mejora de la ganadería mestiza de doble propósito. Maracaibo, Venezuela: Astro Data. p 398-412.

19. Meeusen E. 1999. Immunology of helminth infections, with special reference to immunopathology. Vet Parasitol 84: 259-273.

20. Morales G. 1989. Epidemiología y sinecología de los helmintos parásitos de ovinos y caprinos de zonas áridas del estado Lara, Venezuela. Rev Fac Cienc Vet, UCV 36: 9-52.

21. Morales G, Pino LA, Sandoval E, Moreno $L$. 1998. Relación entre la carga parasitaria, las especies del orden Strongylida presentes y el número de huevos en heces de caprinos naturalmente infectados. Vet Trop 23: 101-107.

22. Morales G, Pino LA, Sandoval E, Moreno L, Jiménez D, Balestrini C. 2001. Dinámica de los niveles de infestación por estróngilos digestivos en bovinos a pastoreo. Parasitol al Día 25: 115-120.

23. Morales G Pino LA. 2003. Carga parasitaria de nemátodos gastrointestinales y la riqueza específica en ovinos naturalmente infectados. Vet Arg 20 (192): 100-108.

24. Morales G, Pino LA, Sandoval E, Florio J, Jiménez D. 2006a. Niveles de infestación parasitaria, condición corporal y valores de hematocrito en bovinos resistentes, resilientes y acumuladores de parásitos en un rebaño Criollo Río Limón. Zootecnia Trop 24: 333-346. 
25. Morales G, Pino LA, Sandoval E, Florio J, Jiménez D. 2006b. Niveles de infestación parasitaria, condición corporal en bovinos resistentes doble propósito infestados en condiciones naturales. REDVET 7(4). [Internet]. Disponible en: http://veterinaria.org/revistas/ redvet $/ n^{\circ} 040406 . h t m l$

26. Morales G, Sandoval E, Pino LA, Balestrini C, García F. 2007. El control de la infestación por estróngilos digestivos en rumiantes domésticos bajo principios de la "Agricultura de Precisión”. REDVET 8(8). [Internet]. Disponible en: http://www.veterinaria.org/revistas/redvet $/ \mathrm{n}^{\circ} 080807 / 080716 . p d f$

27. Morales G, Sandoval E, Pino LA, Rondón Z. 2008. Evaluación de dos criterios de utilidad en un programa de control de la infección por nematodos gastrointestinales en ovinos mediante tratamiento antihelmíntico selectivo. Zootecnia Trop 26: 141-150

28. Morales G Pino LA. 2009. Nematodes parásitos de los rumiantes domésticos en Venezuela. Diagnóstico y control. Caracas: Dot Print. 142 p.

29. Morales G Guillén A, Pinho A, Pino L, Barrios F. 2010. Clasificación por el método Famacha y su relación con el valor de hematocrito y recuento de hpg de ovinos criados en condiciones de pastoreo. Zootecnia Trop 28(4): 545-555.

30. Moreno L. 1996. Helmintosis gastrointestinal bovina. Epidemiología y control en Venezuela. Tópicos sobre Parasitología Veterinaria. Maracay: Pfizer-Salud Animal. p 9-22.

31. O’Kelly J. 1980. Parasitism and blood composition in genetically different types of cattle grazing in a tropical environment. Vet Parasitol 6: 381-390.

32. Peña M, Millar J, Wyatt W, Kearney M. 2000. Differences in susceptibility to gastrointestinal nematode infection between Angus and Brangus cattle in south Louisiana. Vet Parasitol 89: 51-61.
33. Ramírez L. 2005. La condición corporal: Tecnología para mejorar la eficiencia reproductiva del rebaño vacuno. Mundo Pecuario 1(3): 58-59.

34. Romero J. 2005. ¿Qué clase de problemas merecen intervención del veterinario en el campo? Red de Helmintología para América Latina y el Caribe. Conferencia Electrónica. [Internet]. Disponible en: http://cnia.inta.gov.ar/helminto

35. Sandoval E, Espinoza E, González N, Morales G, Montilla W, Jiménez D. 1998. Encuesta serohematológica en bovinos tripanosusceptibles de dos unidades agro-ecológicas del Valle de Aroa. Rev Científica FCV-LUZ 8: 235-238.

36. Sandoval E, Morales G, Pino LA, Jiménez D. 2006. El ganado criollo Río Limonero y su resistencia al parasitismo gastrointestinal. Venezuela Bovina 21(71): 30-32.

37. Strong L. 1993. Overview: the impact of avermectins on pastureland ecology. Vet Parasitol 48: 3-17.

38. Urquhart GM, Armour J, Duncan JL, Dunn AM, Jennings FW. 1999. Veterinary parasitology. $2^{\text {nd }}$ ed. Glasgow: Blackwell Sciences. 307 p.

39. Valle A. 2008a. Bioclimatologia tropical vacuna. Maracay: Ind Gráfica Integral. 509 p.

40. Valle A. 2008b. Vacunos en América. Instituto Nacional de Investigaciones Agrícolas. Centro Nacional de Investigaciones Agropecuarias. Publicación Especial $N^{\circ} 24$, Maracay, Venezuela. 326 p.

41. VanWyk J. 2001. Refugia - overlooked as perhaps the most potent factor concerning the development of anthelmintic resistance. Ondestepoort J Vet Res 68: 55-67.

42. Vercruysse J, Dorny P. 1999. Integrated control of nematode infections in cattle: A Reality? A need? A future? Int J Parasitol 29: 165-175. 